\title{
How New Caledonian crows solve novel foraging problems and what it means for cumulative culture
}

\author{
Corina J. Logan ${ }^{1,5}$ • Alexis J. Breen ${ }^{2} \cdot$ Alex H. Taylor ${ }^{3} \cdot$ Russell D. Gray $^{3}$ • \\ William J. E. Hoppitt ${ }^{4,6}$
}

Published online: 15 August 2015

(C) Psychonomic Society, Inc. 2015

\begin{abstract}
New Caledonian crows make and use tools, and tool types vary over geographic landscapes. Social learning may explain the variation in tool design, but it is unknown to what degree social learning accounts for the maintenance of these designs. Indeed, little is known about the mechanisms these crows use to obtain information from others, despite the question's importance in understanding whether tool behavior is transmitted via social, genetic, or environmental means. For social transmission to account for tool-type variation, copying must utilize a mechanism that is action specific (e.g., pushing left vs. right) as well as context specific (e.g., pushing a particular object vs. any object). To determine whether crows can copy a demonstrator's actions as well as the contexts in which they occur, we conducted a diffusion experiment using a novel foraging task. We used a nontool task to eliminate any confounds introduced by individual differences in their prior tool experience. Two groups had demonstrators (trained in
\end{abstract}

Electronic supplementary material The online version of this article (doi:10.3758/s13420-015-0194-x) contains supplementary material, which is available to authorized users.

Corina J. Logan

c1417@cam.ac.uk

1 SAGE Center for the Study of the Mind, University of California Santa Barbara, Santa Barbara, CA, USA

2 School of Biology, University of St Andrews, St Andrews, UK

3 School of Psychology, University of Auckland, Auckland, New Zealand

4 Animal and Environment Research Group, Department of Life Sciences, Anglia Ruskin University, Cambridge, UK

5 Present address: Department of Zoology, University of Cambridge, Cambridge, UK

6 Present address: School of Biology, Leeds University, Leeds, UK isolation on different options of a four-option task, including a two-action option) and one group did not. We found that crows socially learn about context: After observers see a demonstrator interact with the task, they are more likely to interact with the same parts of the task. In contrast, observers did not copy the demonstrator's specific actions. Our results suggest it is unlikely that observing tool-making behavior transmits tool types. We suggest it is possible that tool types are transmitted when crows copy the physical form of the tools they encounter.

Keywords New Caledonian crow $\cdot$ Social learning $\cdot$ Learning mechanisms · Information transmission $\cdot$ Cumulative technological culture

New Caledonian crows (Corvus moneduloides) are one of the few species that make and use tools in the wild (Hunt, 1996; Hunt \& Gray, 2004). Tool types differ across the crows' geographic range. For example, crows cut the edges off of Pandanus plant leaves to make narrow, wide, and stepped tools for digging into holes in logs to fish out grubs (Hunt \& Gray, 2003, 2004). What causes and maintains tool-type variation is unknown. One possibility is that tool designs are socially transmitted within groups through social learning, and changes in tool designs accumulate across generations (cumulative technological culture hypothesis; Hunt \& Gray, 2003). This would constitute a case of nonhuman animal "culture" (Allen, Weinrich, Hoppitt, \& Rendell, 2013; Aplin et al., 2015; Hunt \& Gray, 2003; Laland \& Hoppitt, 2003). A second possibility is that differences in behavior might solely be a result of different genetic predispositions in each group; for example, some isolated hand-raised juvenile New Caledonian crows make and use tools without observing the behavior of demonstrators (Hunt, Lambert \& Gray 2007; 
Kenward, Weir, Rutz, \& Kacelnik, 2005). However, other New Caledonian crows do not automatically make and use tools, and additional experiments indicate that inherited abilities and social learning likely interact to produce the complex tool manufacture and use observed in the wild (Kenward et al., 2005; Kenward, Rutz, Weir, \& Kacelnik, 2006). A third possibility is that each group's local ecology shapes its behavior in different ways via asocial learning (Laland \& Janik, 2006). For example, in another tool-making and tool-using bird species, the woodpecker finch of the Galapagos, individuals living in more unpredictable environments develop tool-use behavior regardless of whether they observe others using tools (Tebbich, Taborsky, Fessl, \& Blomqvist, 2001; Tebbich, Taborsky, Fessl, \& Dvorak, 2002). Taken together, these results illustrate that the social transmission of tool designs and asocial learning about what makes a more functional tool remain key unexplored factors that could explain variation in New Caledonian crow tool types.

Obtaining direct evidence for the cumulative technological culture hypothesis is difficult: Ideally, to rule out the genetic and ecological alternatives, translocation experiments would be required, which are impractical and ethically questionable for New Caledonian crows (Laland \& Hoppitt, 2003). An alternative approach comes from the suggestion by Kenward and colleagues (2006), who posit that imitation or emulation is required to explain the crows' regional variation in tool types. If this is the case, then studies that assess whether New Caledonian crows are capable of social learning by using mechanisms that could support the social transmission of different tool designs could provide indirect evidence for the cumulative technological culture hypothesis. Imitation involves copying the motor pattern required to make a specific tool and thus could explain the social transmission of specific tool designs (Hoppitt \& Laland, 2013). Emulation generally refers to cases when an observer attempts to recreate the results of a demonstrator's behavior rather than copying the behavior directly (Holzhaider, Hunt, \& Gray, 2010b; Hoppitt \& Laland, 2008, 2013; Tomasello, 1990), though emulation could take a number of specific forms (Whiten, Horner, Litchfield, \& Marshall-Pescini, 2004; see Discussion). In addition to these mechanisms, local (Thorpe, 1956) and stimulus (Spence, 1937) enhancement could also be used. Local enhancement is where one individual's behavior attracts an observer to a specific location and leads the observer to learn about objects at that location. Stimulus enhancement occurs when one individual's behavior attracts an observer's attention to a specific type of stimulus, making the observer more likely to respond to, or interact with, stimuli of that type in the future.

Many other mechanisms have been postulated to play a role in social learning, often with subtle distinctions between alternative mechanisms, making them difficult to distinguish empirically (Hoppitt \& Laland, 2013). To resolve this issue, Hoppitt and Laland (2013) suggest that mechanisms underlying learning by observation can be usefully divided using three key features that are relatively easy to detect empirically: (1) the mechanism allows copying that is action specific: The specific actions used by the demonstrator are transmitted (like imitation and emulation); (2) the mechanism is context specific: It can result in transmission of behavior that is only performed in a specific context, such as at a specific location (like local enhancement) or in response to a particular class of stimuli (like stimulus enhancement); and (3) the mechanism is sensitive to the outcome of the demonstrator's actions (e.g., rewarded behavior is more likely to be transmitted than unrewarded behavior). Further subdivisions may then be made, such as whether context specificity is specific to a location (e.g., local enhancement) or to a particular class of stimuli (e.g. stimulus enhancement). However, Hoppitt and Laland (2013) argue that the key features used in their classification characterize the conditions most commonly presented in experimental studies of social learning mechanisms.

Hoppitt and Laland's (2013) simplified system suits our purposes well, since the first two features capture the necessary properties a social learning mechanism must have to support variation in tool form: The mechanism must be both context specific and action specific. A mechanism that is only context specific (e.g., local or stimulus enhancement) could facilitate tool-making behavior by attracting crows to Pandanus leaves and making them more likely to interact with the leaves. However, mechanisms that are only context specific cannot account for the transmission of specific tool types among birds. This is because different tool types are constructed from the same materials: It is the actions used to process these materials that determines a tool type, so the mechanism must be action specific for the tool type to be transmitted (Kenward et al., 2006, make a similar point).

In this study, we assessed whether New Caledonian crows use social learning mechanisms that could support the social transmission of different tool designs. We presented a novel, nontool foraging task to three groups of wild-caught crows in an open group diffusion experiment. By analyzing the spread of different task solution behaviors through each group, we determined whether the social learning mechanisms used were action specific (e.g., imitation or emulation) as well as context specific (e.g., location or stimulus specific). We also assessed whether the mechanism was sensitive to the outcome of the demonstrator's actions (e.g., whether rewarded behavior was more likely to be transmitted than unrewarded behavior). Translated to a tool-using context, individuals that observe others obtain food with tools might be more likely to attend to the actions performed by the demonstrator, thereby facilitating the transmission of tool types. Individuals were free to interact with one another and the task, a situation that more closely reflects social learning opportunities in the wild than a dyadic demonstrator-observer experiment in which the 
experimenter tightly controls the observational experience of the subjects (Hoppitt \& Laland, 2013; Whiten \& Mesoudi, 2008). We recorded who observed whom interacting with which option on the apparatus, for how long, whether they successfully obtained the food, the latency to interact with each access option, the duration of interaction, and whether food was obtained. Our dynamic analytical method allowed us to investigate the degree to which multiple social and asocial learning mechanisms act and interact (c.f. Hoppitt, Samson, Laland, \& Thornton, 2012), and thus quantify the relative importance of each in how crows solve this novel foraging task.

We modified a commonly used two-action social learning apparatus to understand which learning mechanisms the crows used. Often, two-action apparatuses have only one locus with, for example, a door that can be pushed to the left or right (e.g., Aplin, Sheldon, \& Morand-Ferron. 2013; Aplin et al., 2015; Fawcett, Skinner, \& Goldsmith, 2002; Zentall, Sutton, \& Sherburne, 1996). However, without at least one additional locus in a separate location on the apparatus (e.g., Heyes \& Saggerson, 2002) and at least two replicates of the same apparatus (e.g., Hoppitt et al., 2012), one cannot distinguish among a greater number of learning mechanisms. We made two additional loci on our apparatus, which allowed us to distinguish local enhancement (observers attend to the general area of the apparatus) from imitation/emulation (observers attend to the demonstrator's actions at the two-action locus). We also placed two replicates of the same apparatus on the testing table to distinguish between stimulus enhancement (observers attend to the stimulus they observed the demonstrator interact with, regardless of which apparatus the demonstrator was at) and local enhancement (observers attend to any stimulus on the apparatus the demonstrator interacted with).

\section{Method}

Fourteen New Caledonian crows were caught in the wild in May and June 2013 and temporarily housed in outdoor aviaries on Grand Terre, New Caledonia (Electronic Supplementary Material [ESM] 1). Aviaries and testing rooms were $2.5 \mathrm{~m}$ wide by $3 \mathrm{~m}$ high by $4-5 \mathrm{~m}$ long, mostly covered in shade cloth, with the top partially covered by a metal roof. Birds were fed dog food, papaya, and meat, and had ad libitum access to water at all times.

\section{Task design}

Each of the two social learning apparatuses had three loci for accessing food (hard-boiled eggs). One locus had two methods for accessing the same food container, giving a total of four different options for solving the task (see Fig. 1). Locus 1 had a two-action access mechanism (e.g., Aplin et al., 2013,

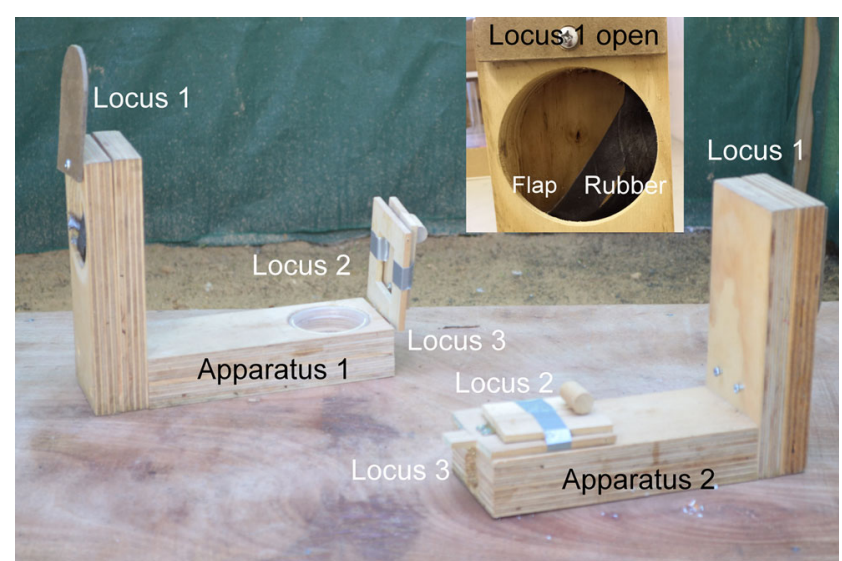

Fig. 1 The two identical apparatuses placed on the table as they were in the experiment with the three loci labeled on each apparatus. Options on the left apparatus are open to show the food compartments, and a close-up of Locus 1 is inset to show what is exposed when swiveling the door to the left or right

2015): The food could be accessed by pushing a swiveling door from the left to the right and putting the bill in the food compartment ("Vflap" option) or by pushing the same swiveling door from the right to the left and poking the bill through a piece of rubber to access the same food compartment ("Vrubber" option). The two-action mechanism at Locus 1 allowed us to examine whether crows imitate or emulate motor actions because we added two other loci at different locations on the apparatus. At Locus 2, food could be obtained by lifting up a wooden flap ("Hflap" option), and at Locus 3, food was obtained by inserting the bill or a tool through a hole in the side of the apparatus ("Hside" option) that accessed the same food cup as Hflap.

The task design allowed us to determine whether any social learning mechanisms were in operation during the foraging sessions (Hoppitt \& Laland, 2013). If a context-specific mechanism was operating on a sufficiently small scale, we would expect an observer to be attracted to the same locus at which they observed an interaction, and to generalize between the two methods that could be used at Locus 1 since both were directed to the same location (e.g., observation of Vflap on Apparatus 1 would have an effect on both Vflap and Vrubber on Apparatus 1). The experimental design (i.e., having two identical apparatuses on the table next to each other) also enabled us to investigate whether context specificity was specific to a location or whether the effect further generalized to the equivalent location on the other apparatus, as would be expected by stimulus enhancement (e.g., observation of Vflap at Apparatus 1 would generalize to Vflap/Vrubber on both Apparatuses 1 and 2). If an action specific mechanism was operating, an observer would be more likely to use the same option they saw demonstrated (e.g., we would expect observation of Vflap at Apparatus 1 to affect Vflap interactions, but not Vrubber interactions; Table 1 shows the pattern of 
Table 1 Pattern of generalization assumed for the social effects in the Cox model. Context specific (CS) mechanisms (e.g., stimulus enhancement) would result in the pattern of generalization represented by all shaded cells (gray and black), whereas action specific (AS) mechanisms (e.g., imitation) would be specific to each option (black cells only). See data at the KNB Data Repository for a description of task options

\begin{tabular}{|c|l|l|l|l|l|}
\cline { 3 - 6 } \multicolumn{2}{c|}{} & \multicolumn{4}{c|}{ Social effect on: } \\
\cline { 2 - 6 } \multicolumn{2}{c|}{} & \multicolumn{3}{c|}{ Vertical } & \multicolumn{2}{c|}{ Horizontal } \\
\hline \multirow{2}{*}{ Observed interaction: } & Flap & Rubber & Flap & Side \\
\hline \multirow{2}{*}{ Locus } & Option & & & & \\
\hline \multirow{2}{*}{1} & Vertical Flap & & & & \\
\cline { 2 - 6 } & Vertical Rubber & & & & \\
\hline 3 & Horizontal Flap & & & & \\
\hline
\end{tabular}

generalization corresponding to each class of social learning mechanisms).

\section{Diffusion experiment}

There were two experimental groups, each with a demonstrator trained in isolation to solve a particular option (demonstrators demonstrated different options) on either of the two identical apparatuses, and a third group (the control group) that had no trained demonstrator. The demonstrator was then released into a group aviary where the experiments were conducted. The first group consisted of four adults (two mated pairs): B and G, YR and OO. In this group (hereafter the B group), the demonstrator (B) was trained over the course of 3 days to solve the Vflap option at Locus 1; however, this demonstrator ended up demonstrating the Hside option at Locus 3 when the experiment began. To ensure demonstrations of both the horizontal and vertical sections of the apparatus occurred in our experiment, the demonstrator (WO) in the second group (hereafter the WO group) was trained over the course of 4 days to solve the Vflap option at Locus 1. WO demonstrated the option she was trained on. The WO group consisted of one adult (W) and five juveniles (WO, WR, BO, WLB, and WB). The control group (hereafter C group) had no trained demonstrator and consisted of a mated pair ( $\mathrm{R}$ and $\mathrm{RG}$ ) and their two offspring (Y and YG). The last four sessions did not include $\mathrm{R}$ because he died. Additionally, any individual that was observed interacting with the apparatus during an experiment was considered a demonstrator, and this experience was accounted for in the analysis. To allow for our lack of control over individual observational experience, we used a statistical modeling approach where each individual's interactions and/or successes with the task were modeled as a function of their prior experience observing other individuals, allowing us to quantify the influence, if any, of each social learning mechanism.
Demonstrator training sessions were carried out in a testing aviary where the demonstrators were visually isolated from other crows and trained on Vflap by closing all other options on the apparatus with tape and taping the flap open to show the food. As the bird became comfortable putting its head in the hole, the flap tape was removed so the bird could learn how to move the flap to access the food. After birds began accessing the food on their own, they were required to successfully access the food on five consecutive trials and then pass a 1-trial field test in which all tape was removed such that all options were available. The two apparatuses were placed on the table, and the bird had to demonstrate the food-access method they were trained on.

Eight experimental sessions were conducted in the testing aviary for each of three groups, spaced 12 to 72 hours apart, ranging from 11 to 45 minutes in duration per session (B group $=206 \mathrm{~min}$ total, $\mathrm{WO}$ group $=360 \mathrm{~min}$ total, control group $=164 \mathrm{~min}$ total; see a video of the experiment at https:// www.youtube.com/watch? $v=60 V F 11 \mathrm{SLwHs})$. Sessions were carried out in a testing room with two identical foraging-task apparatuses oriented in opposite directions, spaced $30 \mathrm{~cm}$ apart on a table $(153 \times 61 \times 75 \mathrm{~cm})$, and recorded with a Nikon D5100 camera (see Fig. 1). Birds in each group were placed in a testing room together. Sessions ended after $45 \mathrm{mi}-$ nutes or when there was no bird on the table for 60 to $70 \mathrm{sec}$ onds (unless they were actively looking for material to bring to the table to solve the task).

Birds that interacted with the apparatus and the birds that observed these interactions were recorded by watching the videos in QuickTime Player v. 10.3 and entering the data in iWork'09 Numbers v. 3.2. Interactions were coded by the locus and option chosen (Locus 1: Vflap or Vrubber, Locus 2: Hflap, Locus 3: Hside), including the start and stop times of the interaction, whether observers saw the demonstrator obtain food or interact with the apparatus without obtaining food, and which apparatus was interacted with (left or right; see Table 1). A bird was considered to have observed another interacting with an apparatus if it was at or above the height of the table in the testing room or located on the ground far 
enough away from the table such that they could see the apparatuses on top.

Dominance behavior (displacements, threats, and conflicts) that occurred on the experimental table was coded for the first four sessions per group to determine the rank order. However, in the case of the control group, which consisted of one family with already established dominance relationships, there were so few aggressive interactions that aggression across all eight sessions was included in the analysis. The dominance rank of each individual within its group was calculated as the total number of aggressive interactions initiated divided by the total number of aggressive interactions engaged in (initiated + received).

\section{Statistical analysis}

Our approach combined elements of diffusion models developed by Hoppitt et al. (2012); Atton, Hoppitt,
Webster, Galef, and Laland (2012); and Hobaiter et al. (2014; see ESM2, Section B4). We first analyzed the data to infer the social influences on the time at which each crow first attempted to solve the task using each of the four options. We used a Cox proportional hazards model, stratified by group such that the analysis was sensitive only to the order in which events occurred within each group: This means that any external influences that differed between groups cannot confound the analysis, even if they varied over time. The form of the Cox model we used is sensitive to similarities in times of solving of any option within each group. For example, if one group all attempted Vflap first and another group all attempted Hside first, this would be taken as evidence of different options spreading through each group by social transmission. The full model specifies the rate of first attempt at method $l$ at locus $k$ for individual $i$ in group $j$ at time $t$ as:

$$
\lambda_{i j k l}(t)=\lambda_{0, j}(t) \exp \left(O_{k l}+\varphi_{i j}+\beta_{L S} L S_{i j k}(t)+\beta_{L G} L G_{i j}(t)+\beta_{C S} C S_{i j k l}(t)+\beta_{A S} A S_{i j k l}(t)\right)\left(1-z_{i j k l}(t)\right),
$$

where $\lambda_{0, j}(t)$ is an unspecified baseline function assumed to be the same for all of group $j$ across all options; $O_{k l}$ is a parameter allowing for differences in difficulty between the four options, with $O_{11}=0$ set as baseline; $\varphi_{i j}$ is a linear predictor containing individual-level variables representing sex, age (adult vs. juvenile), dominance rank, and a random effect allowing for multiple events from the same individual. $L S_{i j k}(t)$ (locationspecific learning) is a binary variable allowing for the fact that having attempted one method at Locus 1 might affect the rate at which the other method is first attempted, either due to generalization of learning between methods at the same location, or in case knowledge of one method inhibits learning the other. We also included a similar effect, $L G_{i j}(t)$, that generalized across all four options: Learning one option might promote or inhibit learning of the other three. $\beta_{X}$ are fitted parameters, each giving the effect of a variable $X ; z_{i j k l}(t)$ takes the value 1 if $i$ has previously interacted with locus $k$ using method $l$, or if $i$ was a seeded demonstrator for that option, and is 0 otherwise. The $\left(1-z_{i j k l}(t)\right)$ thus ensures that the model only models the rate of first interaction using each option. The remaining terms model social influences on learning, which we now define.

We initially included continuous variables representing a context-specific effect $\left(C S_{i j k l}(t)\right.$, henceforth "CS") and an action-specific effect $\left(A S_{i j k l}(t)\right.$, henceforth "AS"), such as imitation or emulation. The AS variable was the number of successful interactions using method $l$ at locus $k$ observed by individual $i$ prior to $t$, so modeled a social learning effect that was specific to an option. The CS variable was a similar effect that generalized between actions directed toward the same stimulus (i.e., the same specific locus on the apparatus). Since Vflap and Vrubber were directed to the same locus on the apparatus, we assumed a CS effect would generalize between them, whereas Hflap and Hside were directed to distinct loci, so we assumed that a CS effect would distinguish between them (see Table 1 for a diagrammatic representation of the modeled social effects).

CS and AS assumed a social effect in which each successive observation of another crow interacting with the task had the same (multiplicative) effect on the rate of interaction. However, it could be that a single observation is sufficient for a sizeable effect on behavior. For example, a single observation of another crow interacting with the vertical loci may be enough to attract an observer to that location, with later observations having relatively little influence. To allow for this possibility, we considered two corresponding binary variables, $\dot{C} S$, and $\dot{A} S$ (i.e., $\dot{C} S=1$ when $\mathrm{CS}>0$ and 0 otherwise, etc.). Use of the binary variables resulted in an improved model fit (see ESM2, Section B1). Consequently, in the results we report an analysis including the binary $C \dot{C}$, and $A S$ variables (see ESM2, Section B1, for full model specification).

We also wished to test whether the social learning mechanisms in operation were sensitive to the outcome of the demonstrator's actions (i.e., did an observer need to see an interaction that resulted in successful extraction of food, or was an unsuccessful interaction sufficient for an effect to occur?). 
Consequently, we also fitted models in which $\dot{C} S$ and $A ́ S=1$ when a successful interaction at the relevant locus had been observed, and was 0 otherwise (i.e., both when no interactions had been observed and when only unsuccessful interactions had been observed), and compared the fit with models in which an unsuccessful manipulation was sufficient for the effect to occur.

For all analyses we used a model averaging approach using Akaike's Information Criterion corrected for sample size $\left(\mathrm{AIC}_{\mathrm{c}}\right.$; Burnham \& Anderson, 2002), allowing us to extract Akaike weights quantifying the total support for each variable, model-averaged estimates of effect size, and confidence intervals that allowed for model-selection uncertainty. We ran an equivalent analysis looking for social influences on the rate at which crows solved the task using each option once they had first attempted that option (see ESM2, Section B3). Analyses were conducted in the R Statistical Environment v. 3.1.0 (R Core Team, 2014) using the coxme (Therneau, 2012), lme 4 (Bates, Maechler, Bolker, \& Walker, 2014) and MuMIn (Bartoń, 2014) packages.

\section{Data availability}

Data used in the analyses and a description of the behavior at each locus is available at the KNB Data Repository (Logan \& Hoppitt, 2015).

\section{Ethics statement}

This research was carried out in accordance with the University of Auckland's Animal Ethics Committee (permit number R602).

\section{Results}

There were dominance hierarchies within each group, with two exceptions: WB's rank was unknown because he sat on the side throughout testing, and therefore we ranked him last in the group; R's rank was also unknown because he did not participate in aggressive interactions even though he was an active member of the group, and therefore we ranked him in the middle to minimize the influence this data had on the model fit (see ESM1, Table A1).

Table 2 gives the support for each variable in the analysis of the rate of interaction, along with model averaged estimates and confidence intervals. There was strong support for a contextspecific effect of observation with $86 \%$ total support for the corresponding binary variable (ĆS; see Table 2 and Fig. 2). The context specific effect was due to stimulus enhancement rather than local enhancement (see Fig. 3, and see further explanation in ESM2, Section B2). Crows that had observed another crow interacting with the task at a specific locus were an estimated 5.3 times faster (see Note below) to start interacting with the task at that locus, $95 \%$ unconditional CI $[1.25,22.3]$. There was no evidence that additional observations of interactions at a locus further increased the rate of interaction at that locus (AICc increased by 1.67). Taken together, these results suggest a smallscale context-specific effect, whereby crows are more likely to interact with stimuli they have seen other crows interacting with, and that this effect only requires a single observation to manifest itself. In contrast, there was little evidence of an action specific (AS) effect consistent with imitation or emulation (total support $=38 \%$ ). (Note: Cox survival analysis model the rates at which events of a specific type occur as a function of the predictor variables for each individual. These rates then determine the probability a particular individual/event type combination will be the next to occur, thus allowing the model to be fitted to data

Table 2 Summary analysis of effects on the rate of interaction using each option

\begin{tabular}{lll}
\hline Variable/effect & Support (total Akaike weight) & $\begin{array}{l}\text { Back-transformed multiplicative effect } \\
(95 \% \text { unconditional confidence interval) }\end{array}$ \\
\hline Context-specific observation effect (e.g., stimulus enhancement) & $86 \%$ & $5.3 x(1.25-22.3)$. \\
Action-specific observation effect (e.g., imitation/emulation) & $38 \%$ & $2.19 x(0.36-13.4)$ \\
Option & $97 \%$ & Relative to Hflap: \\
& & Hside: $1.35 x(0.5-3.60)$ \\
& & Vflap: $0.57 x(0.22-1.48)$ \\
Locus-specific asocial effect & & $0.94 x(0.34-2.55)$ \\
Locus-general asocial effect & $20 \%$ & $0.35 x(0.06-2.24)$ \\
Sex (males-females) & $25 \%$ & $5.8 x(0.99-33.6)$ \\
Age (adults-juveniles) & $74 \%$ & $0.96 x(0.27-3.42)$ \\
Rank & $22 \%$ & $1.70 x(0.99-2.90)$ per rank position \\
\hline
\end{tabular}

Note. For interpreting Akaike weights, note that $p<0.05$ in a likelihood ratio test with $1 d f$ corresponds to an Akaike weight of $>72 \%$ in favor of the more complex model. 

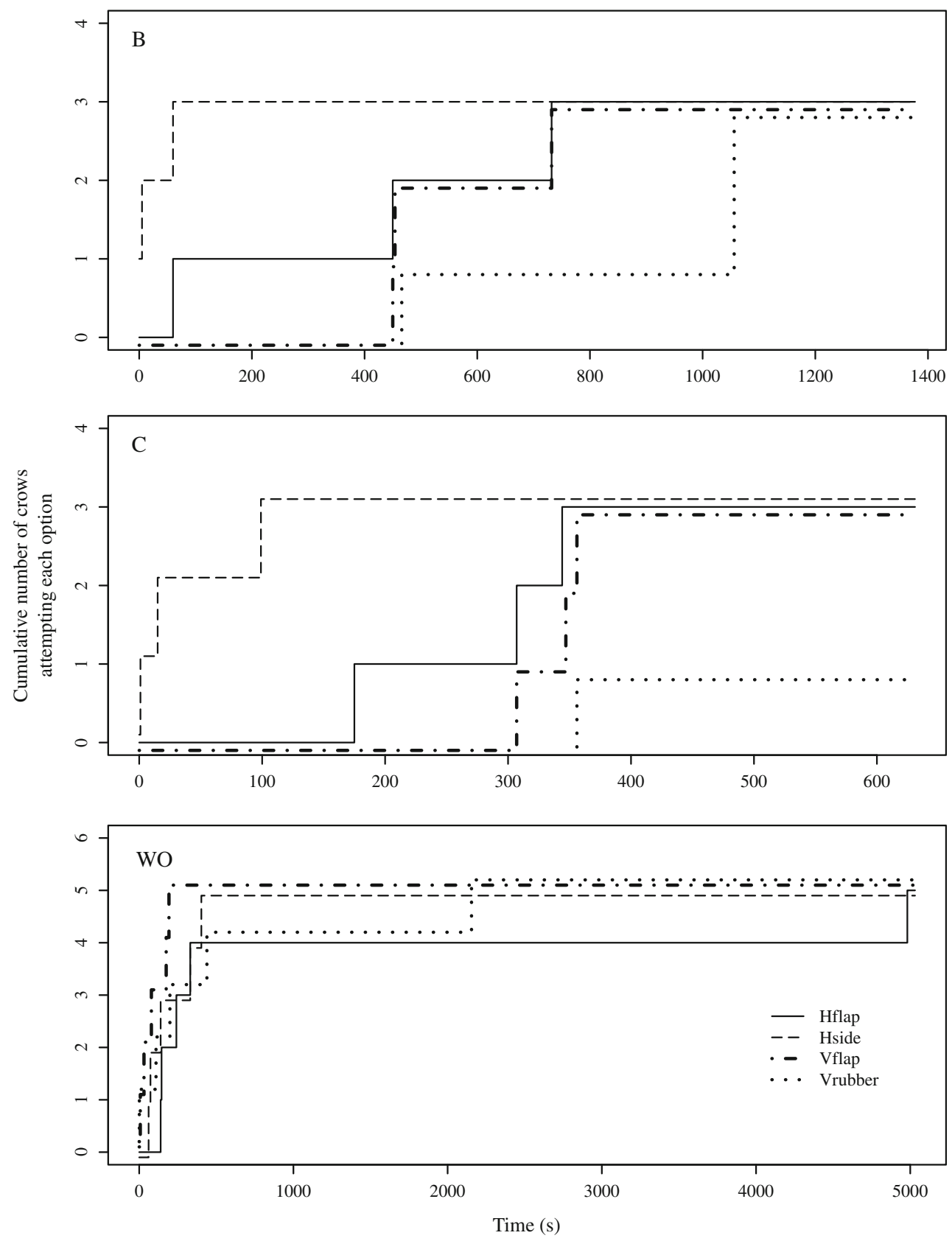

Fig. 2 Diffusion curves for each option in each group (B, C, WO). Within each group, crows start attempting to solve the task using a given option at a relatively similar time, consistent with social learning

giving the order in which events occurred. Thus, we are able to estimate the effect of each variable in terms of how much faster or slower the relevant events occur.)

There was strong evidence of an underlying difference in interaction rate among the four options (total support $=97 \%$; see Table 2) and little evidence that learning to interact with the task using one method at Locus 1 generalized to or inhibited interaction using the other method at that locus (total support $=20 \%$ ). Likewise, there was little evidence that learning to interact using one option had an effect on the other three triggered by an initial "innovation." However, while Hflap (Locus 2) and Hside (Locus 3) are triggered independently in each group, Vflap and Vrubber (both directed to Locus 1) are triggered as one

options (total support $=25 \%$ ). There was some evidence of an effect of sex (support $=74 \%$ ) with males being an estimated 5.8 times faster to attempt each option, $95 \%$ CI [0.99, 33.6], and of rank (support $=64 \%$ ) with higher ranked individuals being faster to attempt each option: An estimated effect of 1.7 times per rank position, $95 \%$ CI $[0.99,2.9]$. There was little evidence for an effect of age (support $=22 \%$ ). However, the confidence intervals are broad for these variables, being based on a small sample for comparing individuals $(n=14$; see Table 2). 


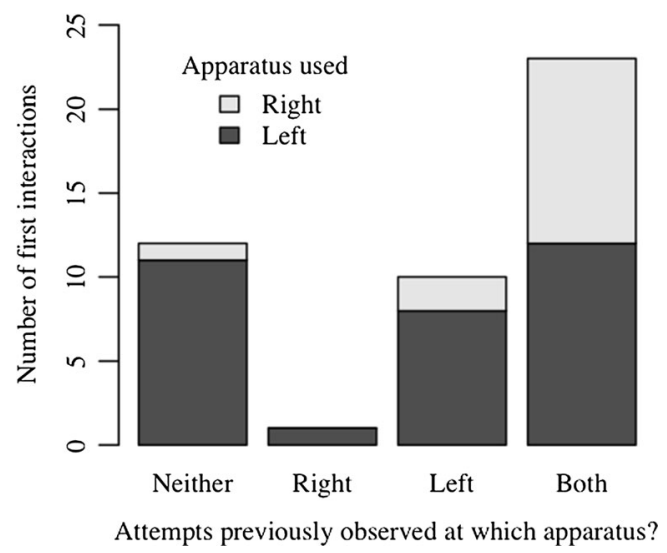

Fig. 3 The apparatus used for first attempts at each locus, broken down by whether an interaction using that locus had previously been observed at the left apparatus, the right apparatus, neither, or both

We also could not accurately estimate the difference in the (binary) stimulus enhancement effect between adults and juveniles. This effect is estimated to be 1.13 times stronger in juveniles, but with $95 \%$ UCI $[0.25,5.22]$ : so a sizeable difference in either direction remains plausible. However, we can clearly conclude that the stimulus enhancement effect is not restricted to juveniles or to adults. When we constrain the effect to be zero for adults in the best model, $\mathrm{AIC}_{\mathrm{c}}$ increased by 6.7 , corresponding to 29.1 times more support for a model where adults are affected by observing others. Likewise, when we constrain the effect to be zero for juveniles, $\mathrm{AIC}_{\mathrm{c}}$ increased by 5.5 , corresponding to 15.5 times more support for a model where juveniles are affected by observing others. We have clear evidence that the stimulus enhancement effect operates on both adults and juveniles, but we are unable to say with confidence which age class is affected more strongly.

We found weak evidence that the CS effect was sensitive to the outcome of the observed individuals' actions, since models in which observation of an unsuccessful interaction with locus $k$ was sufficient for the CS effect to occur had slightly less support (0.62 times) than models where observation of a successful interaction was required (see ESM2, Section B2). However, we found no evidence that the choice of apparatus was influenced by the apparatus at which the interactions of others were observed, suggesting the CS effect generalized between apparatuses, as expected if stimulus enhancement was operating, and was not specific to a location, as expected if local enhancement was operating (see Fig. 3 and ESM2, Section B2).

There was no evidence that observation had any influence on how quickly the crows solved the task using a specific option once they first interacted with that option (support $<23 \%$ in all cases). It therefore appears that social learning acts to attract crows to specific stimuli associated with the task (the loci), but there is no evidence that they socially learn anything about how to successfully manipulate the apparatus to obtain food. There was weak evidence that lower ranked crows were faster to solve the task using a particular option once they started using that option (support $=56 \%$ ), with an estimated increase of 1.47 times per unit decrease in rank, $95 \%$ UCI $[0.95,2.27]$. All other variables in the model had little support $(<42 \%)$.

\section{Discussion}

We found strong evidence that wild-caught juvenile and adult New Caledonian crows used a social learning mechanism that is context specific, but not action specific, to acquire information about a novel foraging task, and then used trial and error learning to solve the task. Observers who saw a demonstrator succeed in obtaining food at a particular locus had an increased likelihood of attempting to solve the task using that locus relative to other loci. However, the effect generalized between different actions for solving the task that were directed to the same locus, therefore they did not use the same actions they observed others using to solve that locus. Furthermore, after their first attempt to solve the task using a specific option, observations of others attempting or succeeding using that option did not decrease their latency to success using that option. This suggests that they used trial and error learning to converge on the actions required to solve the task at each locus, rather than copying the actions they observed others using.

The context-specific effect we detected is consistent with both stimulus enhancement and observational conditioning since both result in the same pattern of generalization between options. Stimulus enhancement predicts that observing another crow's interactions with a particular locus draws the observer's attention to that locus, and thus makes them more likely to interact with it (potentially on both apparatuses). Alternatively, it could be that observation resulted in crows learning an association between a particular locus and food when they observed a conspecific extracting food from that locus (observational conditioning; sensu Heyes, 1994), thus causing the observer to interact with that locus sooner (again, potentially on both apparatuses). Observational conditioning of this kind would be sensitive to the outcome of the demonstrator's actions, as we and others (Akins \& Zentall, 1998) have found, since an association is only likely to form if the demonstrator is successful in extracting food from the locus in question. However, it is also possible that a successful interaction is simply more effective at attracting an observer's attention to a stimulus. In contrast, a small-scale local-enhancement effect, whereby observation of an interaction with a locus on a specific apparatus would attract observers to that specific location, is unlikely to account for our results. We found no evidence that the choice of apparatus was influenced by the apparatus at which the interactions of others were observed suggesting the context specific effect generalized between apparatuses, as would be expected by stimulus enhancement, but not local 
enhancement (see ESM2, Section B2). Whilst the task did not involve tool making, we assume that any social learning mechanism found to play a role in the acquisition of novel foraging behavior is also likely to play a role in the acquisition of tool-making behavior.

Since action-specific social learning mechanisms (e.g., imitation or emulation) would be required to account for the documented pattern of variation in New Caledonian crow tool types, that we found no action-specific effect in our diffusion experiment suggests that social learning resulting from observing another's tool-making activity is unlikely to explain tool type variation. It is possible that New Caledonian crows are capable of action-specific social learning, but that they only use it to copy tool-making behavior and not foraging behavior in general. While this seems unlikely, further experiments will be required to rule out this possibility.

Nonetheless, our results suggest it is unlikely that tool types are transmitted among crows by observation of tool making. This does not completely rule out the possibility that tooltypes are socially transmitted, since it is possible that New Caledonian crows learn which tool type to make by copying the physical products or artifacts of other crows' tool-making behavior (the tools themselves) as suggested by Holzhaider, Hunt, and Gray (2010a, 2010b). We term this the "tool template matching hypothesis." Just as young songbirds learn a mental template of their species song and match their developing song to the template (Doupe \& Konishi, 1991; Konishi, 1985; Nottebohm, 1984;), so New Caledonian crows might form a mental template of their parent's tools, through using their parent's tools during development, and/or by observing the counterparts (cut outs left on the leaves) of tools left in Pandanus plants. Tool-template matching would be a form of emulation (and thus be action specific without necessarily directly observing the actions of another) since the crows are recreating the results of another individual's behavior. However, rather than recreating object movements resulting from a demonstrator's actions after having observed those movements and actions directly, a specific tool shape would be imprinted during development and then recreated via trial and error learning (see Fig. 4).
There are a number of documented cases of social learning via the products or artifacts of another individual's behaviour (e.g., Terkel, 1996; Thornton \& McAuliffe, 2006), though, as Fragaszy and colleagues (2013) argue, the role artifacts play in the maintenance of technical traditions, such as tool use, in nonhuman animals, has been largely overlooked. In most cases, it is likely that artifacts indirectly influence the behavior of another in a manner that leads to their learning a skill by attracting their attention to a relevant location (local enhancement) or by providing the opportunity to practice that skill (Caro \& Hauser, 1992; Hoppitt et al., 2008). A recent experiment investigated tool behavior in Goffin's cockatoos, who are not reported to use tools in the wild, finding that they learned to make and use tools by emulating the results of the demonstrator's actions rather than the demonstrator's action sequence (Auersperg et al., 2014). This suggests that result emulation might be a more dominant learning mechanism than previously thought. In contrast, the tool template-matching hypothesis states that New Caledonian crows can directly copy the products they encounter, something that, to our knowledge, has not been demonstrated in nonhuman animals, and may require specialized cognitive abilities. Consequently, testing the hypothesis seems a promising route for further research into the factors influencing the emergence of cumulative culture.

Although the context specific mechanisms we found in operation cannot account for the transmission of specific tool types, we suggest it is plausible that these mechanisms play a role in the acquisition of tool-related behavior in the wild. Juveniles often observe parents using Pandanus tools, giving abundant opportunities to draw their attention to the tool itself by context specific mechanisms like stimulus enhancement (Holzhaider et al., 2010b). Furthermore, parents often leave their tools in cavities and juveniles pick them up and try to use them (Holzhaider et al., 2010b). However, young crows rarely observe their parents making tools, suggesting that opportunities to imitate or emulate the actions used to make the tool are limited (Gray, personal observation). Furthermore, tool template matching by itself, if it occurs, is unlikely to be very effective at encouraging the learning of tool-related behaviors

\section{Development of behavior}

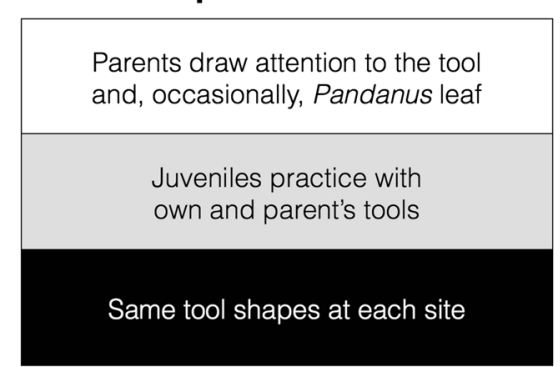

Fig. 4 The elementary tool-related behavior observed in the field that has been proposed to lead to cumulative technological culture (Holzhaider et al., 2010b; Hunt \& Gray, 2003) can be explained by the learning

\section{Learning mechanisms}

Stimulus Enhancement: What to attend to
Anyone draws attention to an option

Trial \& Error Learning: How to solve

After first try, further observations of solves

does not decrease latency to solve

Imprint: What shape

Repeatedly see and use parent's tool,

which is a particular shape

mechanisms found in our lab study. The final step in this pathway, Imprint, is hypothetical, requiring experiments for validation 
because juveniles may be unlikely to encounter and recognize discarded tools and/or counterparts without having their attention attracted to those objects by another crow's manipulations of those objects. However, their strong propensity for contextspecific social learning suggests that, after observing others obtain food with tools, observers will be more likely to seek out and interact with discarded tools that visually resemble those they saw others using.

Our finding that both juveniles and adults were socially influenced by observing others leads us to question previous assumptions that (1) there is a sensitive period during which learning about foraging occurs, and (2) learning is restricted to vertical transmission (e.g., parents to offspring). It has been proposed that juveniles may make tool shapes more similar to their parents' than to other conspecifics by paying more attention to their parents than to others (Holzhaider et al., 2011). However, given our results, this effect could simply be a result of juveniles being exposed to their parent's tool shapes much more than to other tool shapes, thus biasing what tool shape they copy. Therefore, social dynamics in the wild could constrain crows' learning. Indeed, New Caledonian crows live in extended family groups (St Clair et al., 2015; Holzhaider et al., 2011) and there is evidence that they come into close proximity with neighboring groups when resources are abundant, though the nature of these interactions is unknown (Rutz et al., 2012; St Clair et al., 2015). The context specific effect we identify in our experiment could also play a role in maintaining family specific tool "lineages": though family groups can interact, crows are likely to form a template of tools and/or counterparts they have had more exposure to (i.e., the tools of those with whom they most frequently interact).

In conclusion, our new evidence weighs against the hypothesis that imitation or emulation following observation of toolmaking behavior explains the pattern of variation in tool form observed in New Caledonian crows. Assessment of the alternative tool template-matching hypothesis requires further experiments directly evaluating the evidence that exposure to a specific tool form, under the appropriate social conditions, strongly influences the probability that a crow will learn to make tools of the same form. If such evidence is found, the case for cumulative culture in New Caledonian crows would be greatly strengthened, and cast doubt on the notion that imitation and teaching are necessary for cumulative culture to evolve.

Acknowledgments We thank Dieter Lukas and Kevin Laland for discussions; Joe Jablonski for apparatus design ideas and for co-constructing the social learning apparatus; Christin Palmstrom for the photos and videos demonstrating the apparatus option actions; and Alecia Carter, Neeltje Boogert, Hannah Rowland, and two anonymous reviewers for manuscript feedback. We are grateful to our funders: the SAGE Center for the Study of the Mind at the University of California Santa Barbara and the National Geographic Society/Waitt Grants Program (CJL), a Rutherford Discovery Fellowship from the Royal Society of New Zealand (AHT), the Marsden Fund (RG), and a BBSRC grant (WH;

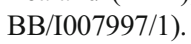

Data accessibility Data on the study subjects is in ESM1; the data set on which the models were run is available at the KNB Data Repository (Logan \& Hoppitt 2015: https://knb.ecoinformatics.org/\#view/doi:10. 5063/F1JH3J44), a video demonstrating the apparatus options is available at figshare.com (http://dx.doi.org/10.6084/m9.figshare. 1480629), and a video showing the experiment is at YouTube (https:// www.youtube. $\mathrm{com} /$ watch? $\mathrm{v}=60 \mathrm{VF} 11 \mathrm{SLwHs})$.

\section{References}

Akins, C. K., \& Zentall, T. R. (1998). Imitation in Japanese quail: The role of reinforcement of demonstrator responding. Psychonomic Bulletin \& Review, 5, 694-697.

Allen, J., Weinrich, M., Hoppitt, W., \& Rendell, L. (2013). Networkbased diffusion analysis reveals cultural transmission of lobtail feeding in humpback whales. Science, 340, 485-488.

Aplin, L. M., Farine, D. R., Morand-Ferron, J., Cockburn, A., Thornton, A., \& Sheldon, B. C. (2015). Experimentally induced innovations lead to persistent culture via conformity in wild birds. Nature, 518, 538-541. doi:10.1038/nature13998

Aplin, L. M., Sheldon, B. C., \& Morand-Ferron, J. (2013). Milk bottles revisited: Social learning and individual variation in the blue tit (Cyanistes caeruleus). Animal Behavior, 85, 1225-1232.

Atton, N., Hoppitt, W., Webster, M. M., Galef, B. G., \& Laland, K. N. (2012). Information flow through threespine stickleback networks without social transmission. Proceedings of the Royal Society B, 279, 4272-4278. doi:10.1098/rspb.2012.1462

Auersperg, A. M. I., von Bayern, A. M. I., Weber, S., Szabadvari, A., Bugnyar, T., \& Kacelnik, A. (2014). Social transmission of tool use and tool manufacture in Goffin cockatoos (Cacatua goffini). Proceedings of the Royal Society B, 281, 20140972. doi:10.1098/ rspb.2014.0972

Bartoń, K. (2014). MuMIn: Multi-model inference (R package version 1.10.0). Available at http://CRAN.R-project.org/package=MuMIn. Accessed 1 Oct 2014.

Bates, D., Maechler, M., Bolker, B., \& Walker, S. (2014). lme4: Linear mixed-effects models using Eigen and S4 (R package version 1.1-6). Available from http://CRAN.R-project.org/package $=1$ me 4 . Accessed 1 Oct 2014.

Burnham, K. P., \& Anderson, D. R. (2002). Model selection and multimodel inference: A practical information-theoretic approach. New York, NY: Springer.

Caro, T. M., \& Hauser, M. D. (1992). Is there teaching in nonhuman animals? The Quarterly Review of Biology, 67, 151-174.

Doupe, A. J., \& Konishi, M. (1991). Song-selective auditory circuits in the vocal control system of the zebra finch. Proceedings of the National Academy of Sciences-Biology, 88, 11339-11343.

Fawcett, T. W., Skinner, A. M., \& Goldsmith, A. R. (2002). A test of imitative learning in starlings using a two-action method with an enhanced ghost control. Animal Behavior, 64, 547-556.

Fragaszy, D. M., Biro, D., Eshchar, Y., Humle, T., Izar, P., Resende, B., \& Visalberghi, E. (2013). The fourth dimension of tool use: Temporally enduring artefacts aid primates learning to use tools. Philosophical Transactions of the Royal Society, Series B, 368. doi: 10.1098/rstb.2012.0410

Heyes, C. M. (1994). Social learning in animals: Categories and mechanisms. Biological Reviews, 69, 207-231. doi:10.1111/j.1469-185X. 1994.tb01506.x

Heyes, C., \& Saggerson, A. (2002). Testing for imitative and nonimitative social learning in the budgerigar using a two-object/two-action test. Animal Behavior, 64, 851-859.

Hobaiter, C., Poisot, T., Zuberbuhler, K., Hoppitt, W., \& Gruber, T. (2014). Social network analysis shows direct evidence for social 
transmission of tool use in wild chimpanzees. PLoS Biology, 12, e1001960. doi:10.1371/journal.pbio.1001960

Holzhaider, J. C., Hunt, G. R., \& Gray, R. D. (2010a). The development of Pandanus tool manufacture in wild New Caledonian crows. Behaviour, 147, 553-586. doi:10.1163/ $000579510 X 12629536366284$

Holzhaider, J. C., Hunt, G. R., \& Gray, R. D. (2010b). Social learning in New Caledonian crows. Learning \& Behavior, 38, 206-219. doi:10. 3758/LB.38.3.206

Holzhaider, J. C., Sibley, M. D., Taylor, A. H., Singh, P. J., Hunt, G. R., \& Gray, R. D. (2011). The social structure of New Caledonian crows. Animal Behavior, 81, 83-92. doi:10.1016/j.anbehav.2010.09.015

Hoppitt, W. J., Brown, G. R., Kendal, R., Rendell, L., Thornton, A., Webster, M. M., \& Laland, K. N. (2008). Lessons from animal teaching. Trends in Ecology \& Evolution, 23, 486-493.

Hoppitt, W., \& Laland, K. N. (2008). Social processes influencing learning in animals: A review of the evidence. Advanced in the Study of Behavior, 38, 105-165.

Hoppitt, W., \& Laland, K. N. (2013). Social learning: An introduction to mechanisms, methods, and models. Princeton, NJ: Princeton University Press.

Hoppitt, W., Samson, J., Laland, K. N., \& Thornton, A. (2012). Identification of learning mechanisms in a wild meerkat population. PLoS ONE, 7, e42044. doi:10.1371/journal.pone.0042044

Hunt, G. (1996). Manufacture and use of hook-tools by New Caledonian crows. Nature, 379, 249-251. doi:10.1038/379249a0

Hunt, G. R., \& Gray, R. D. (2003). Diversification and cumulative evolution in New Caledonian crow tool manufacture. Proceedings of the Royal Society B, 270, 867-874. doi:10.1098/rspb.2002.2302

Hunt, G. R., \& Gray, R. D. (2004). The crafting of hook tools by wild New Caledonian crows. Proceedings of the Royal Society, B, 271, S88-S90. doi:10.1098/rsbl.2003.0085

Hunt, G. R., Lambert, C., \& Gray, R. D. (2007). Cognitive requirements for tool use by New Caledonian crows (Corvus moneduloides). New Zealand Journal of Zoology, 34, 1-7. doi:10.1080/ 03014220709510058

Kenward, B., Rutz, C., Weir, A. A. S., \& Kacelnik, A. (2006). Development of tool use in New Caledonian crows: Inherited action patterns and social influences. Animal Behavior, 72, 1329-1343. doi:10.1016/j.anbehav.2006.04.007

Kenward, B., Weir, A. A. S., Rutz, C., \& Kacelnik, A. (2005). Tool manufacture by naive juvenile crows. Nature, 433, 121. doi:10. $1038 / 433121 \mathrm{a}$

Konishi, M. (1985). Birdsong: From behaviour to neuron. Annual Review of Neuroscience, 8, 125-170.

Laland, K. N., \& Hoppitt, W. (2003). Do animals have culture? Evolutionary Anthropology, 12, 150-159. doi:10.1002/evan.10111

Laland, K. N., \& Janik, V. M. (2006). The animal cultures debate. Trends in Ecology \& Evolution, 21, 542-547. doi:10.1016/j.tree.2006.06. 005
Logan, C., \& Hoppitt, W. (2015). Social learning in New Caledonian crows: A diffusion experiment on 14 wild-caught individuals in aviaries in 2013. The Knowledge Network for Biocomplexity. doi: 10.5063/F1JH3J44

Nottebohm, F. (1984). Birdsong as a model in which to study brain processes related to learning. Condor, 86, 227-236.

$\mathrm{R}$ Core Team. (2014). $R$ : A language and environment for statistical computing. Vienna, Austria: R Foundation for Statistical Computing. Available at http://www.R-project.org/. Accessed 1 Oct 2014.

Rutz, C., Burns, Z. T., James, R., Ismar, S. M., Burt, J., Otis, B., . . St Clair, J. J. (2012). Automated mapping of social networks in wild birds. Current Biology, 22, R669-R671. doi:10.1016/j.cub.2012.06. 037

Spence, K. W. (1937). Experimental studies of learning and higher mental processes in infra-human primates. Psychological Bulletin, 34, 806 850.

St Clair, J. J. H., Burns, Z. T., Bettaney, E. M., Morrissey, M. B., Burt, J., Otis, B., Ryder, T. B., Fleischer, R. C., James, R., Rutz, C. (2015). Experimental resource pulses influence social-network dynamics and the potential for information flow in tool-using crows. Nature Communications. 6, 7197.

Tebbich, S., Taborsky, M., Fessl, B., \& Blomqvist, D. (2001). Do woodpecker finches acquire tool-use by social learning? Proceedings of the Royal Society B, 268, 2189-2193.

Tebbich, S., Taborsky, M., Fessl, B., \& Dvorak, M. (2002). The ecology of tool-use in the woodpecker finch (Cactospiza pallida). Ecology Letters, 5, 656-664.

Terkel, J. (1996). Cultural transmission of feeding behaviour in the black rat (Rattus rattus). In C. M. Heyes \& B. G. Galef (Eds.), Social learning in animals: The roots of culture (pp. 17-47). San Diego, CA: Academic Press.

Therneau, T. (2012). coxme: Mixed effects Cox models (R package version 2.2-3). Available at http://CRAN.R-project.org/package $=$ coxme. Accessed 1 Oct 2014.

Thornton, A., \& McAuliffe, K. (2006). Teaching in wild meerkats. Science, 313, 227-229. doi:10.1126/science.1128727

Thorpe, W. H. (1956). Learning and instinct in animals. London, UK: Hazell Watson and Viney.

Tomasello, M. (1990). Cultural transmission in chimpanzee tool use and signaling. In S. T. Parker \& K. R. Gibson (Eds.), Language and intelligence in monkeys and apes (pp. 274-311). Cambridge, UK: Cambridge University Press.

Whiten, A., Horner, V., Litchfield, C. A., \& Marshall-Pescini, S. (2004). How do apes ape? Learning \& Behavior, 32, 36-52.

Whiten, A., \& Mesoudi, A. (2008). Establishing an experimental science of culture: Animal social diffusion experiments. Philosophical Transactions of the Royal Society B, 363, 3477-3488.

Zentall, T. R., Sutton, J. E., \& Sherburne, L. M. (1996). True imitative learning in pigeons. Psychological Science, 7, 343-346. 